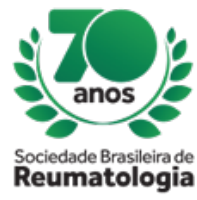

\title{
EVALUATION OF THE INVOLVEMENT OF ANGIOTENSIN II IN THE KIDNEY INJURY CAUSED BY ADJUVANT-INDUCED ARTHRITIS IN RATS
}

Rodrigo Piloto Oliveira Batanero (FACERES Faculdade de Medicina, São José do Rio Preto, SP, Brasil), Glayber Falcão Garcia Filho (FACERES Faculdade de Medicina, São José do Rio Preto, SP, Brasil), João Pedro Lot Doná (FACERES Faculdade de Medicina, São José do Rio Preto, SP, Brasil), Enzo Prandi Carvalho (FACERES Faculdade de Medicina, São José do Rio Preto, SP, Brasil), Euradir Vitório Angeli Junior (FACERES Faculdade de Medicina, São José do Rio Preto, SP, Brasil), Rafael Oliveira Guena (FACERES Faculdade de Medicina, São José do Rio Preto, SP, Brasil), Patricia Maluf Cury (FACERES Faculdade de Medicina, São José do Rio Preto, SP, Brasil), Carla Patrícia Carlos (FACERES Faculdade de Medicina, São José do Rio Preto, SP, Brasil)

\section{BACKGROUND}

Kidney damage caused directly by the rheumatoid arthritis (RA) is not well elucidated, and there is evidence of the involvement of the Renin-Angiotensin System (RAS) in extra-articular manifestations of RA. The objective of the present study was to investigate the renal manifestation of rheumatoid arthritis in rats and the involvement of angiotensin II in the mechanism of the disease.

\section{MATERIALS AND METHODS}

Male Wistar rats (200 g), ingesting low salt diet, were distributed in three groups (8 rats/group): Control, Arthritis and Arthritis + AT1 blocker (losartan). The arthritis was induced by the injection of $100 \mu \mathrm{L}$ of an emulsion of dissected Mycobacterium tuberculosis $(50 \mathrm{mg} / \mathrm{mL}$ ) on the intradermal paw. The control group received the vehicle (mineral oil emulsion). Treatment with losartan was initiated on the first day of immunization by subcutaneous injection daily $(1 \mathrm{mg} / \mathrm{Kg}$ daily). After confirmation of systemic arthritis, the animals were sacrificed 60 days post immunization or vehicle. The following parameters were evaluated: kidney function through the quantification of plasma uric acid, urea and creatinine levels and creatinine clearance; renal injury and inflammation by histopathological analysis and immunohistochemistry for apoptosis (caspase-3), influx of macrophages and TGF- $\beta$ expression; oxidative stress by renal expression of iNOS and plasma TBARS (thiobarbituric acid reactive substances); renal expression of AT1 and AT2 receptors (immunohistochemistry); and plasma concentration of angiotensin II by ELISA assay.

\section{RESULTS}

Compared to control group, arthritic rats showed elevated plasma levels of creatinine, urea, uric acid, TBARS and angiotensin II, as well as reduced creatinine clearance and enhanced kidney macrophage influx and expression of TGF- $\beta$, caspase- 3 and iNOS. The losartan treatment improved these parameters. However, the blockade of the AT1 receptors worsened the inflammation despite the improvement in inflammatory macrophage influx and expression of TGF- $\beta$. There were no differences in renal AT1 and AT2 receptors expression among groups.

\section{CONCLUSION}

Experimental RA causes kidney injury and the pathogenesis of the disease are related to action of angiotensin in renal tissue. Processes FAPESP 2017/18730-6 and FAPERP 067/2018. 